\title{
Mit Selbstverletzungsstörung beim Hautarzt
}

\section{Hier steht eine Anzeige.}

— Wer Hilfe beim ästhetischen Dermatologen sucht, tut dies nicht unbedingt nur für sein Äußeres. „Es geht auch darum, dass der Patient sich innerlich besser fühlt", sagte Prof. Claudia Borelli, Tübingen. Wenn ein Patient unter seinem Aussehen leide und man ihm mit den etablierten Methoden helfen könne, sei das erst einmal völlig in Ordnung, so Borelli. Grenzwertig werde es allerdings, wenn die Beschäftigung mit dem Äußeren überhandnehme. Als Dermatologe müsse man genau austarieren, ob das, was der Patient sich wünsche, objektivierbar sei. Beim Verdacht auf eine körperdysmorphe Störung riet die Expertin zu größter Vorsicht: „Ich fange eine Therapie nur dann an, wenn ich den Eindruck habe, dass ich etwas verbessern kann und dass der Patient sich führen lässt."

Warnsignale, bei denen man besser die Finger von der Behandlung lasse, sei laut Borelli ein im Umgang sehr auffälliger Patient und ein „schlechtes Bauchgefühl“. Das gleiche gelte für frische Narben oder wenn man auf der Haut "überhaupt nicht das sieht, was einem der Patient beschreibt". Hier könne man mit der ästhetischen Dermatologie in keinem Fall gewinnen.

Nicht selten kommen Patienten mit einer selbstverletzenden Störung in die dermatologische Praxis, um die Spuren der Manipulation beseitigen zu lassen. Die leichteste Ausprägung ist dabei die Acne excoriée: Hier werden minimale Hautveränderungen zum Anlass für oft heftigste Manipulationen. Die Patienten leiden nach Borelli oft sehr stark, obwohl die Papeln, Pusteln und Komedonen objektiv gar nicht sehr ins Auge fallen. Charakteristisch sind der Expertin zufolge Hypopigmentierungen: „Hier kann man sehen, dass an der Haut schon viel gearbeitet wurde". Borelli versucht in solchen Fällen zunächst, ein gutes Verhältnis zur Patientin - oft sind es Frauen in psychischen Stresszuständen - aufzubauen, ihr Verständnis entgegenzubringen, sie nicht "herunterzubügeln".

Ein Chemical Peeling sei oft sinnvoll, um das Hautbild zu verbessern. Dabei sei eine ausreichende Analgesie wichtig, um Selbstverletzungstendenzen möglichst wenig zu fördern. Borelli empfiehlt, das „Arbeiten an der Haut" dem Patienten gegenüber sanft zu thematisieren. Im Verlauf der Therapie fragt die Dermatologin etwa, ob es dem Patienten möglich ist, "dieses Arbeiten geistig an mich abzugeben“. Ist der Patient dazu nicht in der Lage, legt Borelli möglichst einfühlsam nahe, psychosomatische Hilfe in Anspruch zu nehmen. Bei stärkeren Ausprägungen wie Selbstverletzungsnarben ist es wichtig zu beurteilen, wie alt diese Artefakte sind. Frische Narben weisen auf einen instabilen Patienten hin. In diesen Fällen rät Borelli, von einer ästhetisch-dermatologischen Therapie abzusehen oder abzuwarten, bis er erfolgreich therapiert wurde. Ältere Narben könne man dagegen durchaus angehen: Vor allem mit dem Microneedling erzielt Borelli in solchen Fällen oft gute Ergebnisse.

"Wenn bei Ihnen die Alarmglocken läuten, sollten Sie nicht behandeln", fasste Borelli zusammen. Vor allem auch, weil beim körperdysmorphen Patienten die Gefahr bestehe, dass man sich zum verlängerten Arm der Selbstverletzung mache. „Die Einsicht muss auf Patientenseite da sein", so die Expertin, "sonst kann man keine Therapie beginnen."

Dr. Elke Oberhofer

Borelli C. Der körperdysmorphe Patient in der ästhetischen Sprechstunde 